\title{
Acute-on-Chronic Liver Failure
}

\author{
Wolf O. Bechstein ${ }^{a} \quad$ Stefan Zeuzem ${ }^{b}$ \\ a Department of General and Visceral Surgery, University Hospital Frankfurt, Goethe University Frankfurt, Frankfurt/M., Germany; \\ ${ }^{b}$ Department of Internal Medicine I, University Hospital Frankfurt, Goethe University Frankfurt, Frankfurt/M., Germany
}

Acute-on-chronic liver failure is a relatively newly defined syndrome which presents with varying clinical scenarios [1]. It is characterized by acute decompensation of a previously known or previously non-diagnosed chronic liver disease leading to organ failure and thus may result in a short-term mortality of $22-73 \%$ depending on the severity of organ failure [2]. To make things even more complicated, acute-on-chronic liver failure is not uniformly defined [3]. However, the need to recognize and adequately treat this entity becomes more pressing as some of the affected patients will already be on the waiting list for a liver transplant. Thus a narrow time window exists where it may be possible to successfully treat these patients and allow for a transplant. Otherwise one may be faced with the fact that the condition of a patient has deteriorated so severely that liver transplantation would seem futile [4].

For the present special topic, we have asked leading scientists and clinicians in this rapidly evolving field to gather the latest data and give their views on the various aspects on acute-on-chronic liver failure, including state of the art of management of the different scenarios with which this syndrome may present.

Appenrodt and Lammert [5] give an overview of the causes and management of renal failure in patients with liver cirrhosis. A nihilistic approach is not warranted since new classifications and biomarkers allow for a more differentiated approach compared to the past.

Coagulopathy remains a challenge since bleeding - often from esophageal varices - may precipitate acute-on-chronic liver failure and may be compounded by excessive blood loss. In addition, invasive diagnostic and therapeutic procedures in this setting require at least temporary normalization of coagulation. Drolz et al. [6] not only highlight the underlying pathophysiological mechanisms but also list practical recommendations.

Infections may also precipitate and complicate acute-onchronic liver failure. Both community-acquired and nosocomial infections present challenges since overt sepsis has a grim prognosis and usually presents a contraindication to liver transplantation. Engelmann and Berg [7] elegantly describe the approach to infection in these scenarios.
One of the more groundbreaking views on acute-on-chronic liver failure is the consideration of the 'gut-liver-axis' which links portal hypertension to this multi-faceted syndrome. This includes not only diagnostic but also therapeutic implications as is shown in detail by Trebicka et al. [8].

Another innovative view is the analysis of immunodysfunction which accompanies or predisposes to the development of acute-onchronic liver failure. Lange and Moreau [9] elucidate how more and more knowledge about these pathophysiological mechanisms is emerging while the therapeutic implications are not yet fully realized.

Due to the nature of acute-on-chronic liver failure, afflicted patients will usually be admitted to an intensive care unit (ICU). Based not only on current literature but also on their immense personal experience, Katsounas and Canbay [10] offer practical advice on how to manage various aspects of organ failure in these patients.

As liver transplantation may be the only hope for a successful outcome while on the other hand the severity of organ failure may lead to a futile transplant outcome, the decision whether to transplant or de-list the patient becomes particularly pressing. Heise et al. [11] present a systematic approach to decision making which may be helpful when dealing with patients, relatives, and also colleagues who may have unrealistic expectations with regard to liver transplantation.

Finally, an expert panel of experienced clinicians in this field was brought together by Lange [12] who compiled a set of detailed questions to confront both hepatologists and transplant surgeons - thus an interesting spectrum of answers has emerged which illustrates that this is still a field of uncertainty how to deal with this growing clinical challenge in present and future.

We hope that this special topic will be helpful for readers and contribute to both timely recognition of acute-and-chronic liver failure and its various facets and also to implement up-to-date therapeutic interventions and thus eventually save lives.

\section{KARGER}

(c) 2018 S. Karger GmbH, Freiburg

Fax +497614520714
Prof. Dr. med. Wolf Otto Bechstein 


\section{References}

1 Bernal W, Jalan R, Quaglia A, Simpson K, Wendon J, Burroughs A. Acute-on-chronic liver failure. Lancet 2015;386:1576-1587.

2 Arroyo V, Moreau R, Jalan R, Gines P. Acute-onchronic liver failure: a new syndrome that will re-classify cirrhosis. J Hepatol 2015;62:S131-S143.

$\checkmark 3$ Duseja A, Singh SP. Toward a better definition of acute-on-chronic liver failure. J Clin Exp Hepatol 2017;7:262-265.

4 Olson JC, Karvellas CJ. Critical care management of the patient with liver cirrhosis awaiting liver transplant in the intensive care unit. Liver Transplantation 2017; 23:1465-1476.

5 Appenrodt B, Lammert F: Renal failure in patients with liver cirrhosis: novel classifications, biomarkers, treatment. Visc Med 2018;34: DOI: 10.1159/000492587.
Drolz A, Ferlitsch A, Fuhrmann V: Management of coagulopathy during bleeding and invasive procedures in patients with liver failure. Visc Med 2018;34: DOI: 10.1159/000491106.

7 Engelmann C, Berg T: Management of infectious complications associated with acute-on-chronic liver failure. Visc Med 2018;34: DOI: 10.1159/000491107.

Trebicka J, Reiberger T, Laleman W: Gut-liver axis links portal hypertension to acute-on-chronic liver failure. Visc Med 2018;34: DOI: 10.1159/000490262.

9 Lange CM, Moreau R: Immunodysfunction in acuteon-chronic liver failure. Visc Med 2018;34: DOI: $10.1159 / 000488690$

10 Katsounas A, Canbay A: Intensive care therapy for patients with advanced liver diseases. Visc Med 2018;34: DOI: $10.1159 / 000492088$.
11 Heise M, Weiler N, Iken S, Welker M-W, Zeuzem S, Bechstein WO, Schnitzbauer AA: Liver transplantation in acute-on-chronic liver failure: considerations for a systematic approach to decision making. Visc Med 2018;34: DOI: 10.1159/000492137.

12 Lange CM, Bechstein WO, Berg T, Engelmann C, Bruns T, Canbay A, Moreau R, Trebicka J: Acute-onchronic liver failure. Visc Med 2018;34: DOI: 10.1159/000491406. 